\title{
MMPs in tuberculosis: granuloma creators and tissue destroyers
}

\author{
Padmini Salgame
}

University of Medicine and Dentistry of New Jersey, New Jersey Medical School, Department of Medicine, Center for Emerging Pathogens, Newark, New Jersey, USA.

\begin{abstract}
Most individuals infected with Mycobacterium tuberculosis develop a latent infection, which does not progress to active tuberculosis (TB). This occurs, in part, because infected macrophages recruit immune cells to form a granuloma, isolating the bacteria and preventing its spread. In some individuals, granulomas undergo necrosis and tissue destruction occurs, releasing the bacteria and allowing the development of active disease. In this issue of the $J C I$, Elkington et al. provide evidence that $M$. tuberculosis drives the expression of MMP-1, which in turn promotes the collagen breakdown that leads to alveolar destruction in TB. These findings identify putative therapeutic targets for the prevention of TB.
\end{abstract}

An inimitable feature in the natural history of tuberculosis (TB) is the development of latent infection in individuals exposed to Mycobacterium tuberculosis. Approximately 5\% of those who are latently infected eventually reactivate their latent focus and develop active TB. Infected macrophages in the latently infected host initiate a cascade of events that results in the recruitment of new monocytes from the bloodstream, providing a continuous niche for $M$. tuberculosis to maintain its replicative cycle. As adaptive immunity develops in the host, Th1 cells are recruited to the developing granuloma, and the IFN- $\gamma$ secreted by these cells enhances the antimicrobial capacity of infected macrophages. Thus, the ordered recruitment of monocytes, T cells, B cells, NK cells, and dendritic cells to the granuloma and their positioning around infected macrophages sequesters $M$. tuberculosis, thereby curtailing its spread from the initial foci of infection (1). As the granulomatous response stabilizes, FoxP3 ${ }^{+}$regulatory $\mathrm{T}$ cells are also found in the tubercle lesion (2) and are conceivably recruited to function as gatekeepers of excessive immune response. Some of the bacteria sequestered within this immune structure reprogram their gene expression and enter the latent/persistent phase, with the ability to reactivate years or decades later. The finding that disease reactivation can be significantly reduced by treatment with isoniazid, an antibiotic that targets actively

Conflict of interest: The author has declared that no conflict of interest exists.

Citation for this article: J Clin Invest. doi:10.1172/ JCI57423. growing cells, strongly suggests that there is persistent bacterial replication within the granuloma in latent TB (3). Furthermore, the contribution of the host immune response to the maintenance of granuloma integrity and latent infection is best exemplified in immunosuppressed individuals. Loss of granuloma and the risk of reactivation are significantly increased in HIV-infected individuals and in those treated with TNF- $\alpha$ inhibitors (4). During reactivation, the stable granulomas progress to necrosis and caseation that promotes the release of $M$. tuberculosis into the airways and transmission to new hosts.

\section{MMPs in the spotlight}

With the emergence of new molecular tools and animal models that lend themselves to intravital imaging, the detailed events underlying the structural reorganization of immune cells to form a stable granuloma or progress to a pathologic lesion as the granuloma dissolves are slowly but surely unfolding. Fascinatingly, MMPs are emerging as key players in both the creation of the granuloma and in the destruction of lung tissue.

The first MMP was discovered in 1962 by Gross and Lapiere (5), who demonstrated collagenolytic activity in amphibian tissues undergoing metamorphosis. Since that first report, 24 mammalian MMPs have been identified, with partially overlapping substrate specificities and functions (reviewed in ref. 6). MMPs are zinc-dependent proteases and consist of two conserved domains, a prodomain and a catalytic domain (6). Cooperatively, MMPs can effectively degrade all compo- nents of the extracellular matrix, including collagens, laminin, fibronectin, vitronectin, and proteoglycans. MMP activity is tightly controlled, not only by gene expression, but also by its localization in the cell, its requirement for proenzyme activation, and the concurrent expression of tissue inhibitors (6).

Although the original function ascribed to MMPs was matrix cleavage and tissue remodeling, accumulating evidence suggests that this family of enzymes also plays a major role in angiogenesis, cell motility, apoptosis, and, of particular interest here, regulating immunity, inflammation, and host defenses (reviewed in ref. 7). MMPs also activate intestinal pro- $\alpha$-defensins and function as antimicrobials. Several MMPs, through proteolytic cleavage, can modulate the activity of cytokines and chemokines, including IFN- $\gamma$, IL- $1 \beta$, TNF- $\alpha$, CXCL8, and CCL7. Thus, MMPs can modulate chemokine gradients and regulate leukocyte recruitment to sites of inflammation (reviewed in ref. 7). The role of MMPs in lung biology is gaining attention, and several studies now show that they are important in normal lung function as well as in lung tissue destruction associated with inflammatory lung disorders, including COPD and emphysema (6).

\section{M. tuberculosis holds its host hostage}

M. tuberculosis infection induces the production of MMPs in vitro in macrophages and in vivo in murine lung tissue (8-10). Mice treated with BB-94, a broad-spectrum inhibitor of MMPs, exhibit either a delay in granuloma induction (11) or form smaller granulomas with more collagen (12), suggesting for the first time that MMPs regulate cell migration and granuloma formation after $M$. tuberculosis infection. Consistent with findings using inhibitors, MMP-9-deficient mice also exhibited reduced macrophage recruitment to the lungs and had smaller granulomas (13). These studies provided the basis for the emerging paradigm that MMPs remodel lung tissue and initiate granuloma formation. 


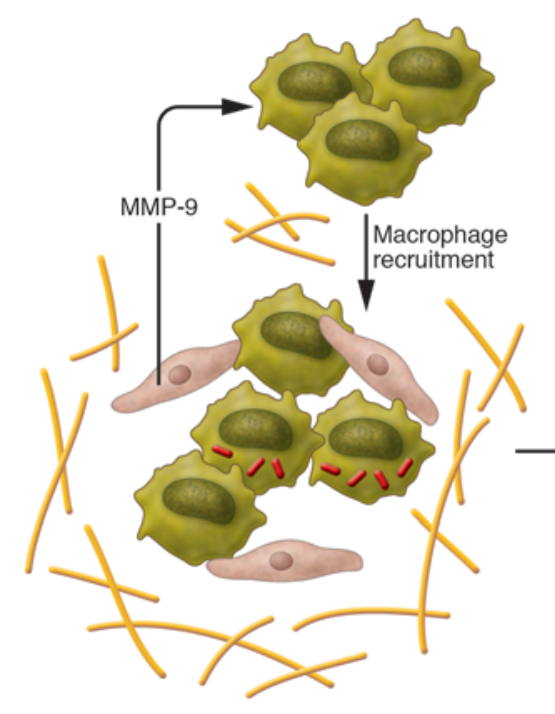

Developing granuloma

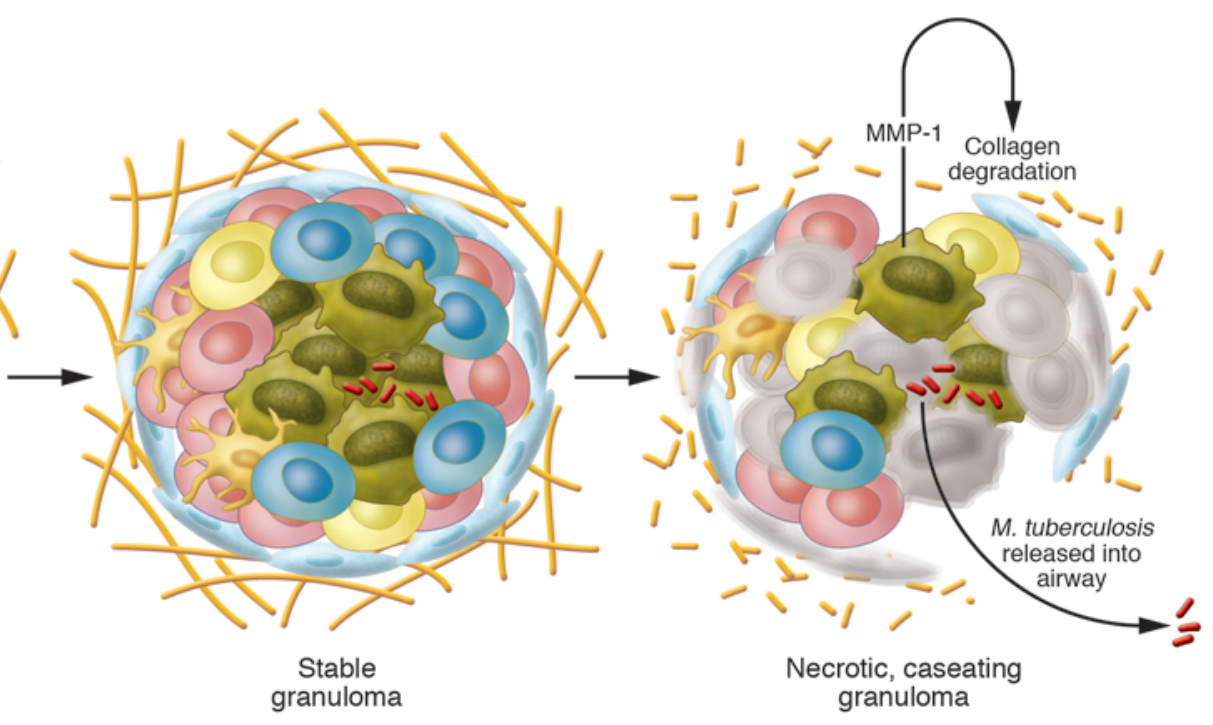

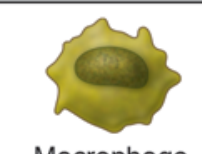

Macrophage

$$
0<0
$$

M. tuberculosis

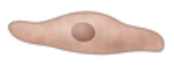

Epithelial cell

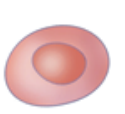

B cell

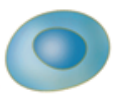

$\mathrm{T}$ cell

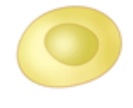

Treg

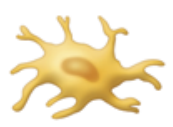

DC

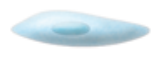

Fibroblast

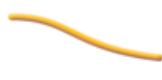

Collagen

\section{Figure 1}

The role of MMPs in the progression of tuberculous granuloma. MMP-9 from epithelial cells initiates recruitment of new monocytes to the developing granuloma. As the granuloma matures, Th1 cells, regulatory T cells, and B cells are recruited to form a stable granuloma, and M. tuberculosis persists in a latent state. During reactivation, granulomas become caseating and necrotic, and excessive MMP-1 secretion from macrophages leads to collagen degradation and tissue destruction, which culminates in M. tuberculosis erosion into the airways.

Subsequently, Ramakrishnan and colleagues (14) discovered the molecular mechanisms that M. tuberculosis uses to induce granuloma formation. They showed that the secreted 6kDa early secreted antigenic target (ESAT-6) protein induced MMP-9 in epithelial cells that were in close proximity to infected macrophages. The upregulation of MMP-9 was associated with the recruitment of newer monocytes and macrophages necessary for granuloma maturation. Knockdown of MMP-9 expression with modified antisense oligonucleotides abrogated granuloma formation and limited bacterial growth, thereby demonstrating the key roles of MMPs in this process. Usurping host MMPs to ensure its long-term latent existence in the host attests to M. tuberculosis's Machiavellian intelligence.

\section{A role for MMPs in progression to TB?}

The hypothesis that $M$. tuberculosis-induced MMPs may also drive tissue destruction and subsequent transmission is suggested by studies that found a correlation between increased MMP levels and severity of disease
$(10,15)$. Moreover, it has been observed that MMP-9 is strongly expressed in monocytes present within TB granulomas that are adjacent to caseous necrosis (16). In this issue of the JCI, Elkington, Friedland, and colleagues provide direct evidence that MMP-1, which can specifically degrade type I collagen, drives destruction of pulmonary tissue in TB (17). The authors profiled the expression of MMPs and tissue inhibitors of metalloproteinases (TIMPs) in induced sputum and bronchoalveolar lavage fluid from TB patients and healthy controls. They found that MMP-1 was significantly upregulated in TB patients while levels of TIMPs were reduced. The mouse MMP-1 ortholog is not expressed in the lung, so to further investigate the role of MMP-1 in lung immunopathology, the authors used a novel transgenic mouse that expresses human MMP-1. They found that M. tuberculosis infection of these mice led to increased MMP-1 expression in the lungs, and this was associated with increased alveolar destruction and significantly greater collagen breakdown in comparison with that of wild-type mice Thus, this study for the first time provides a direct link between MMP-1 and tissue destruction in TB.

Notably, despite extensive tissue destruction in the MMP-1 transgenic mice, granuloma architecture was maintained, and there was no increase in the bacterial burden in the lungs. The authors suggest that this may be due to the fact that necrosis of the granuloma and matrix destruction are two separate events. It is likely that caseous necrosis of granulomas followed by liquefaction and extracellular growth of bacteria occur along with matrix destruction to cause cavities and the subsequent dissemination of viable bacteria into the blood stream or bronchial tree. In the future, this might be addressed by crossing MMP-1 transgenic mice with a mouse strain like $\mathrm{C} 3 \mathrm{HeB} / \mathrm{FeJ}$ that develops necrotic granulomas (18) to develop a model that may closely mimic human tubercle lesions.

Rutter et al. have identified a single nucleotide polymorphism guanine insertion/deletion at $-1,607 \mathrm{bp}$ in the promoter of the human $M M P 1$ gene, which 
results in two alternate alleles (19). The insertion of an additional guanine increases transcriptional activity due to the creation of a core binding site for a transcription factor from the Ets family (19). It would be interesting to test whether this polymorphism favors tissue destruction and enhances risk of disease progression in TB. Supporting this idea, a recent study reported that the presence of the $-1607 \mathrm{G}$ allele was significantly higher in TB patients with advanced pulmonary fibrosis compared with that in those with minimal fibrosis (20).

\section{Conclusions}

Taken together, an emerging model (Figure 1) for the role of MMPs in TB is that M. tuberculosis induces tissue remodeling via induction of MMP-9 to establish itself in the host. Once M. tuberculosis is established within the granuloma, maintaining intact granulomas is beneficial to the host, since it keeps the pathogen under check. During reactivation of a latent infection, excessive MMP-1 secretion leads to matrix degradation and cavitation. Much remains to be learned regarding the spatial and temporal regulation of specific MMPs during lung remodeling in TB. Nonetheless, the work of Freidland's group, along with other recent publications, suggest that MMPs represent viable therapeutic targets to control M. tuberculosis replication soon after infection by preventing nascent granulomas from maturing and by blocking matrix degradation and cavitary disease.

\section{Acknowledgments}

The author's research program is supported by NIH grants AI084822, AI069395, and AI065663.

Address correspondence to: Padmini Salgame, UMDNJ-New Jersey Medical School, Center for Emerging Pathogens, South Orange Avenue, MSB A902, Newark, New Jersey 07101, USA. Phone: 973.972.8647; Fax: 973.972.0713; E-mail: salgampa@ umdnj.edu.

1. Saunders BM, Britton WJ. Life and death in the granuloma: immunopathology of tuberculosis. Immunol Cell Biol. 2007;85(2):103-111.

2. Scott-Browne JP, et al. Expansion and function of Foxp3-expressing $\mathrm{T}$ regulatory cells during tuberculosis. J Exp Med. 2007;204(9):2159-2169.

3. Whalen CC, et al. A trial of three regimens to prevent tuberculosis in Ugandan adults infected with the human immunodeficiency virus. Uganda-Case Western Reserve University Research Collaboration. N Engl J Med. 1997;337(12):801-808.

4. Gardam MA, et al. Anti-tumour necrosis factor agents and tuberculosis risk: mechanisms of action and clinical management. Lancet Infect Dis. 2003;3(3):148-155.

5. Gross J, Lapiere CM. Collagenolytic activity in amphibian tissues: a tissue culture assay. Proc Natl Acad Sci U S A. 1962;48:1014-1022.

6. Parks WC, Shapiro SD. Matrix metalloproteinases in lung biology. Respir Res. 2001;2(1):10-19.

7. Parks WC, Wilson CL, Lopez-Boado YS. Matrix metalloproteinases as modulators of inflammation and innate immunity. Nat Rev Immunol. 2004;4(8):617-629

8. Rivera-Marrero CA, Schuyler W, Roser S, Roman J. Induction of MMP-9 mediated gelatinolytic activity in human monocytic cells by cell wall components of Mycobacterium tuberculosis. Microb Patbog. 2000;29(4):231-244.

9. Quiding-Jarbrink M, Smith DA, Bancroft GJ. Production of matrix metalloproteinases in response to mycobacterial infection. Infect Immun. 2001;69(9):5661-5670.
10. Chang JC, Wysocki A, Tchou-Wong KM, Moskowitz N, Zhang Y, Rom WN. Effect of Mycobacterium tuberculosis and its components on macrophages and the release of matrix metalloproteinases. Tho$\operatorname{rax} .1996 ; 51(3): 306-311$

11. Hernandez-Pando R, Orozco H, Arriaga K, Pavon L, Rook G. Treatment with BB-94, a broad spectrum inhibitor of zinc-dependent metalloproteinases, causes deviation of the cytokine profile towards type-2 in experimental pulmonary tuberculosis in Balb/c mice. Int J Exp Pathol. 2000;81(3):199-209.

12. Izzo AA, Izzo LS, Kasimos J, Majka S. A matrix metalloproteinase inhibitor promotes granuloma formation during the early phase of Mycobacterium tuberculosis pulmonary infection. Tuberculosis. 2004;84(6):387-396.

13. Taylor JL, et al. Role for matrix metalloproteinase 9 in granuloma formation during pulmonary Mycobacterium tuberculosis infection. Infect Immun. 2006;74(11):6135-6144.

14. Volkman HE, Pozos TC, Zheng J, Davis JM, Rawls JF, Ramakrishnan L. Tuberculous granuloma induction via interaction of a bacterial secreted protein with host epithelium. Science. 2010;327(5964):466-469.

15. Hrabec E, Strek M, Zieba M, Kwiatkowska S, Hrabec Z. Circulation level of matrix metalloproteinase-9 is correlated with disease severity in tuberculosis patients. Int J Tuberc Lung Dis. 2002;6(8):713-719.

16. Price NM, Gilman RH, Uddin J, Recavarren S, Friedland JS. Unopposed matrix metalloproteinase- 9 expression in human tuberculous granuloma and the role of TNF-alpha-dependent monocyte networks. J Immunol. 2003;171(10):5579-5586.

17. Elkington P, et al. MMP-1 drives immunopathology in human tuberculosis and transgenic mice. J Clin Invest. 2011;121(5):1827-1833.

18. Pichugin AV, Yan BS, Sloutsky A, Kobzik L, Kramnik I. Dominant role of the sst 1 locus in pathogenesis of necrotizing lung granulomas during chronic tuberculosis infection and reactivation in genetically resistant hosts. Am J Pathol. 2009;174(6):2190-2201.

19. Rutter JL, et al. A single nucleotide polymorphism in the matrix metalloproteinase- 1 promoter creates an Ets binding site and augments transcription. Cancer Res. 1998;58(23):5321-5325.

20. Wang $\mathrm{CH}$, et al. MMP-1(-1607G) polymorphism as a risk factor for fibrosis after pulmonary tuberculosis in Taiwan. Int J Tuberc Lung Dis. 2010;14(5):627-634.

\title{
Fibulin-5: two for the price of one maintaining pelvic support
}

\author{
Gina M. Northington
}

Department of Obstetrics and Gynecology, University of Pennsylvania School of Medicine, Philadelphia, Pennsylvania, USA.

\begin{abstract}
Pelvic organ prolapse (POP) is a disabling disorder in women characterized by a loss of pelvic floor support leading to the herniation of the uterus into or through the vagina. POP is a complex problem that likely involves multiple mechanisms, and available therapies are limited. In this issue of the JCI, Budatha et al. explore the dual role carried out by fibulin-5 in facilitating the assembly of normal elastic fibers and inhibiting MMP-9 activity, revealing a new mechanism critical to the maintenance of pelvic organ support.
\end{abstract}

Conflict of interest: The author has declared that no conflict of interest exists.

Citation for this article: J Clin Invest. doi:10.1172/ JCI57438.
Pelvic organ prolapse (POP) is a major cause of morbidity among women in the United States. It occurs in approximately $35 \%$ of the adult female population, and the incidence increases with age (1). The impact of POP on women is significant, including sexual dysfunction, social isolation, depression, and poor body image (2). The etiology is multifactorial, with a history of vaginal childbirth being the most notable risk factor $(3,4)$. It is estimated that $11 \%-19 \%$ of women will require surgical repair in their lifetime to treat prolapse, and approximately one-third of these will require additional surgery for recurrent disease (5); thus it is important to define the molecular and cellular mecha- 